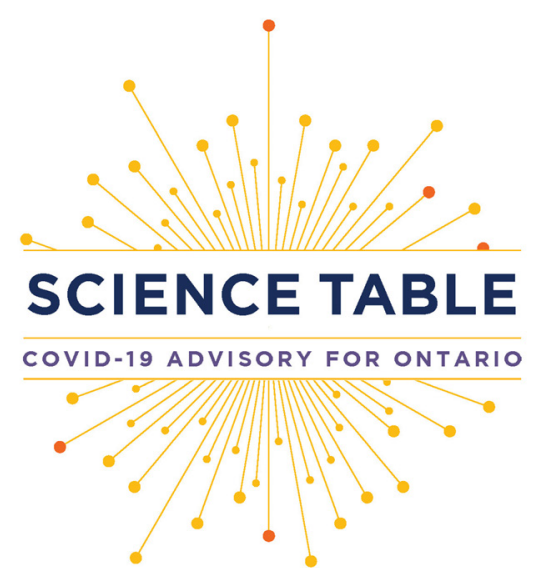

Version: 1.1

Published: May 31, 2021

Updated on June 1, 2021. Version 1.0 is available under Additional Resources at https://doi.org/10.47326/ ocsat.2021.02.33.1.0

Citation: Katz GM, Born KB, Balicer $R D$, et al. Lessons Learned from Israel's Reopening During a Nationwide COVID-19 Vaccination Campaign. Science Briefs of the Ontario COVID-19 Science Advisory Table. 2021;2(33). https://doi.org/10.47326/ ocsat.2021.02.33.1.0

Author Affiliations: The affiliations of the members of the Ontario COVID-19 Science Advisory Table can be found at https:// covid19-sciencetable.ca/.

Declarations of Interest: The declarations of interest of the members of the Ontario COVID-19 Science Advisory Table, its Working Groups, or its partners can be found at https:// covid19-sciencetable.ca/. The declarations of interest of external authors can be found under additional resources at https://doi. org/10.47326/ocsat.2021.02.33.1.0

About Us: The Ontario COVID-19 Science Advisory Table is a group of scientific experts and health system leaders who evaluate and report on emerging evidence relevant to the COVID-19 pandemic, to inform Ontario's response. Our mandate is to provide weekly summaries of relevant scientific evidence for the COVID-19 Health Coordination Table of the Province of Ontario, integrating information from existing scientific tables, Ontario's universities and agencies, and the best global evidence. The Science Table summarizes its findings for the Health Coordination Table and the public in Science Briefs.

Correspondence to: Secretariat of the Ontario COVID-19 Science Advisory Table (info@covid19-sciencetable.ca)

Copyright: 2021 Ontario COVID-19 Science Advisory Table. This is an open access document distributed under the terms of the Creative Commons Attribution License, which permits unrestricted use, distribution, and reproduction in any medium, provided that the original work is properly cited.

The views and findings expressed in this

\section{Lessons Learned from Israel's Reopening During a Nationwide COVID-19 Vaccination Campaign}

Gabrielle M. Katz, Karen B. Born, Ran D. Balicer, Asher Salmon, Kali Barrett, Chaim M. Bell, Yoojin Choi, Laura Desveaux, Gerald A. Evans, Jessica Hopkins, Antonina Maltsev, Allison McGeer, Andrew M. Morris, Anna Perkhun, Fahad Razak, Paula A. Rochon, Brian Schwartz, Arthur S. Slutsky, Tania Watts, Peter Jüni, Nathan M. Stall on behalf of the Ontario COVID-19 Science Advisory Table

\section{Key Message}

Israel maintained an overall decrease in SARS-CoV-2 cases and COVID-19 hospitalizations, ICU admissions, and deaths throughout all phases of reopening.

Key elements of Israel's reopening included a high proportion of adults receiving 2 doses of Pfizer-BioNTech's COVID-19 mRNA vaccine, a phased approach prioritizing the reopening of outdoor activities and schools first, and a 'Green Pass' or vaccination certificate which was introduced from February 21, 2021 to June 1, 2021 to allow fully vaccinated or COVID-19 recovered individuals to enter higher SARS-CoV-2 transmission risk settings.

\section{Summary}

\section{Background}

Ontario's third wave of COVID-19 began on March 1, 2021 and is now under better control with sustained declines in SARS-CoV-2 cases and COVID-19 hospitalizations. The province is moving towards reopening with a three-step plan directed by vaccination rates and health indicators.

As decision-makers continue to plan and implement Ontario's reopening strategy, it is instructive to examine how countries like Israel have successfully executed a phased reopening. The State of Israel has the highest proportion of fully vaccinated individuals in the world. Nearly $62.8 \%$ of all Israelis have received one dose of COVID-19 vaccine, and $59 \%$ have been fully vaccinated as of May 18, 2021. These percentages are based on total population, including children below 16 years of age, who are currently ineligible for vaccination and make up approximately $30 \%$ of Israel's total population.

\section{Questions}

What were the stages of Israel's phased reopening and how were they implemented? What was the observed impact of Israel's phased reopening during a nationwide COVID-19 vaccination campaign on SARS-CoV-2 cases and COVID-19 hospitalizations, ICU admissions, and deaths?

How does Ontario's current SARS-CoV-2 infection rate and COVID-19 vaccination coverage compare to Israel's at the start of its phased reopening?

\section{Findings}

Israel's reopening strategy had four phases which reopened the country from period of lockdown, travel restrictions and broad closures over the course of 6 weeks in February and March 2021. Outdoor spaces opened first, followed by schools, with subsequent 
Science Brief are those of the authors and do not necessarily reflect the views of all of the members of the Ontario COVID-19 Science Advisory Table, its Working Groups, and its partners. loosening of restrictions on indoor gatherings. Israel also introduced 'Green Pass' requirements on February 21, 2021, permitting fully vaccinated or COVID-19 recovered individuals to enter indoor and higher SARS-CoV-2 transmission risk settings. Temporary Green Passes were also granted to children with a negative COVID-19 PCR test within 72 hours. Access to public spaces without a Green Pass gradually expanded with most nonessential establishments required to meet a 'Purple Badge' standard which involved customer and employee COVID-19 symptom screening, specific reductions in capacity, and self-monitored infection prevention measures.

Israel's third lockdown began on January 8, 2021 and was lifted on February 7, 2021 with phase 1 of reopening; at the time of re-opening first and second dose COVID-19 vaccine coverage were $30.1 \%$ and $15.4 \%$ respectively. The nation-wide lockdown drove reductions in SARS-CoV-2 cases and COVID-19 hospitalizations, with sharper declines occurring throughout Israel's phased reopening as vaccine coverage increased. Although children below 16 years of age are not yet eligible for vaccination, a similar drop in SARS-CoV-2 cases has been seen in this age group.

\section{Interpretation}

There are several key takeaways from Israel's successful approach that can inform Ontario's reopening. These include a phased approach beginning with reopening of outdoor spaces, followed by school, and later other indoor spaces and higher SARSCoV-2 transmission risk settings.

Israel's reopening also involved the use of vaccine passports known as the 'Green Pass'. A goal of the Green Pass was to motivate vaccine uptake among younger or vaccine hesitant groups. Full vaccination enabled citizens to hold a green pass, and have access to cultural venues, sports events, and concert halls. When SARS-CoV-2 infection levels were higher, restaurants, hotels, gyms, and other higher transmission risk indoor settings also required a Green Pass to enter. Most non-essential workplaces were required to follow a 'Purple Badge' standard, ensuring the safety and efficiency of workplaces without the need for customer proof of vaccination or COVID-19 recovery. Green Pass and Purple Badge standard requirements expired on June 1, 2021 - corresponding with low nationwide SARS-CoV-2 infection numbers and high COVID-19 vaccine coverage. These were key initiatives to incentivize COVID-19 vaccine uptake and facilitate Israel's safe reopening. The only remaining public health measure is indoor masking. Essential workplaces (such as grocery stores, food markets, transportation, hospitals and pharmacies, police and fire brigades) remained open throughout the lockdown and subsequent reopening phases, and adhered to separate guidelines than the Purple Badge.

The pace of Israel's and Ontario's vaccination campaigns differ substantially. Such differences may be due to land and population size (Ontario has a population 1.6 times greater than Israel, but with 45 times the land area), vaccine implementation (vaccine supply and access), digital infrastructure (centralized in Israel), and timing of the second dose (approximately 21 days in Israel and 120 days in Ontario).

\section{Background}

Ontario's third wave of COVID-19 began on March 1, 2021 and was driven by variants of concern (VOC) - particularly B.1.1.7 - which were associated with a $63 \%$ increased risk of hospitalization, a $103 \%$ increased risk of intensive care unit (ICU) admission and a $56 \%$ increased risk of death due to COVID-19. ${ }^{1}$ The third wave placed tremendous pressure on the province's health care system, with Ontario peaking at 4,812 daily new SARS-CoV-2 cases, with 1,294 COVID-19 patients in hospital, and 661 COVID-19 patients in ICUs on April 16, 2021. 2,3 As of May 19, 2021 there have been a total of 1,545 confirmed COVID-19 deaths during Ontario's third wave. ${ }^{2}$ 
As of mid-May, 2021, public health measures and vaccinations have helped control the transmission in Ontario and driven a reduction in SARS-CoV-2 infected cases, COVID-19 hospitalization and deaths. The 7-day rolling average of SARS-CoV-2 cases has decreased to 2,183 as of May 19, 2021, ${ }^{2}$ while COVID-19 hospitalizations and ICU admissions are also decreasing. Public health measures enacted include a provincewide shutdown of non-essential businesses, schools and other public gathering places on April 3, 2021 and later a province-wide stay-at-home order on April 17, 2021. Ontario's COVID-19 vaccination campaign has also made substantial progress, with more than 53\% of Ontarians having received at least one dose of a COVID-19 vaccine, and approximately $4 \%$ of Ontarians having received two doses as of May 27, 2021. ${ }^{4}$

The Ontario Government released a roadmap on May 20, 2021 outlining a 3-step plan to reopen according to province-wide vaccination rates and health indicators (declining hospitalization, ICU, case rates and percent positivity). ${ }^{5}$ Step one can begin when $60 \%$ of adults have been vaccinated with one dose of a COVID-19 vaccine, provided that there is a continued decline in COVID-19 hospitalizations, ICU occupancy, daily cases and percent test positivity. It will involve a resumption of outdoor activities and small outdoor gatherings. Step two requires that $70 \%$ of adults are vaccinated with one dose and $20 \%$ with two doses, with further reopening of outdoor activities and gatherings, and allowing indoor gatherings of up to five people. Finally, in Step 3,70-80\% of adults must be vaccinated with one dose and $25 \%$ with two doses, allowing for expanded reopening of indoor spaces. In all steps, health indicators will continue to be monitored, and indoor masking will continue to be mandatory in all establishments where it is possible. The province will remain in each step for a minimum of 21 days. Ontario is expected to enter step one of reopening during the week of June 14, 2021 and announced that some outdoor recreational amenities will be reopened on May 22, 2021.

Ontario's reopening plan is aligned with recent Public Health Agency of Canada (PHAC) modelling suggesting that restrictive public health measures closing workplaces, businesses and other public settings could be eased once $75 \%$ of adults have one vaccine dose and $20 \%$ have two. ${ }^{6}$ As decision makers continue planning and implementing the safe reopening of Ontario, it is instructive to examine how countries which are further ahead in the pandemic trajectory have successfully executed a phased reopening strategy. Israel leads the world with the highest proportion of fully vaccinated individuals. ${ }^{7}$ As of May $18,62.8 \%$ of all Israelis have received at least 1 dose of COVID-19 vaccine, and $59.0 \%$ have been fully vaccinated with two doses. ${ }^{8}$ These percentages are based on total population, even though children below 16 years of age - who make up approximately $30 \%$ of Israel's population - are ineligible for vaccination. ${ }^{9}$ Israel enacted phased reopening of the country on February 7, 2021.

\section{Questions}

What were the stages of Israel's phased reopening and how were they implemented?

What was the observed impact of Israel's phased reopening during a nationwide COVID-19 vaccination campaign on SARS-CoV-2 cases and COVID-19 hospitalizations, ICU admissions, and deaths?

How does Ontario's current SARS-CoV-2 infection rate and COVID-19 vaccination coverage compare to Israel's at the start of its phased reopening?

\section{Findings}

\section{Stages of Israel's Phased Reopening}

Israel's third lockdown began on January 8, 2021 during a third wave that was driven by the B.1.1.7 variant, and was lifted on February 7, 2021 with phase 1 of reopening. Unlike Ontario, Israel used a date-based phased approach to reopening, and progressed 
through three additional stages on February 21, March 7, and March 19, 2021. This strategy allowed Israel to reopen public spaces, schools, institutions and businesses, while maintaining some public health measures and minimizing SARS-CoV-2 infections and COVID-19 hospitalizations, ICU admissions, and deaths. Phased reopening began with the reopening of outdoor spaces, directly followed by the gradual reopening of schools (see Table 1).

At the start of the second phase of reopening, 'Green Pass' requirements were introduced to permit only fully vaccinated and COVID-19 recovered individuals with a Green Pass to enter certain establishments (see Tables 1 and 2). The 'Green Pass' was a vaccination certificate or passport that signified that an individual had been fully vaccinated or previously infected and recovered. Upon entering an establishment that requires a Green Pass, vaccinated individuals were required to present their Green Pass along with identification, with the establishment having the option of scanning a falsification-proof QR code on either a smartphone app or a printed piece of paper. The Green Pass aimed to protect high-risk vaccinated individuals and allow them to participate in indoor activities at a time of continued community spread of SARSCoV-2 - since these individuals (older multi-morbid patients) have lower vaccine effectiveness. ${ }^{10,11}$ It was also intended to encourage vaccination uptake, including for those at lower risk of severe COVID-19 disease, by incentivizing entry into socially desirable settings including cultural venues, sports events, and concert halls. ${ }^{12}$ Access to public spaces and activities not requiring a Green Pass has gradually expanded, and Green Pass requirements officially expired on June 1, 2021. Loosening of restrictions on indoor public and private gatherings occurred in the later stages of reopening. Most workplaces were required to meet a 'Purple Badge' standard requiring customer and employee screening, specific reduced capacities, and proper cleaning procedures. These public health measures were also lifted on June 1, 2021 with only universal masking requirements indoors remaining in place. Unlike Ontario, where capacity is often described as percentages, Israel specifies capacity numerous different ways depending on the establishment - including the area of an establishment that can be occupied by one individual, an absolute number, a number based on the amount of staff present in a store, or a percentage.

The country's third lockdown began on January 8, 2021 and included a restriction on residents travelling further than 1000 metres from their place of residence (with some exceptions, such as going to get vaccinated, receive medical treatment, or exercise outdoors) and fines for violating this travel restriction. ${ }^{13}$ Schools and nonessential workplaces were not permitted to be open; however, essential workplaces (for example, grocery stores, public transportation, hospitals and pharmacies, police and fire brigades) followed governmental guidelines for operation throughout the lockdown and subsequent reopening. Israel maintained indoor and outdoor mask mandates during all four phases of reopening, only lifting the outdoor mask mandate on April 18, 2021. ${ }^{14}$ Table 1 provides a summary of each stage of Israel's reopening. 


Reopening Phase
Lockdown Ends, Phase
1 Begins

(February 7, 2021)
Summary of Reopening Phase

- Travel restriction of 1000 metres from home is lifted.

- Outdoor areas including nature reserves, national parks, and sites are opened.

- Businesses with no public facing areas are reopened.

- Children in pre-school, grades 1-4 can go back to school in regions with low SARSCoV-2 infection rates. This was determined based on the rate of new SARS-CoV-2 cases and the percentage of positive test results. ${ }^{15,16}$

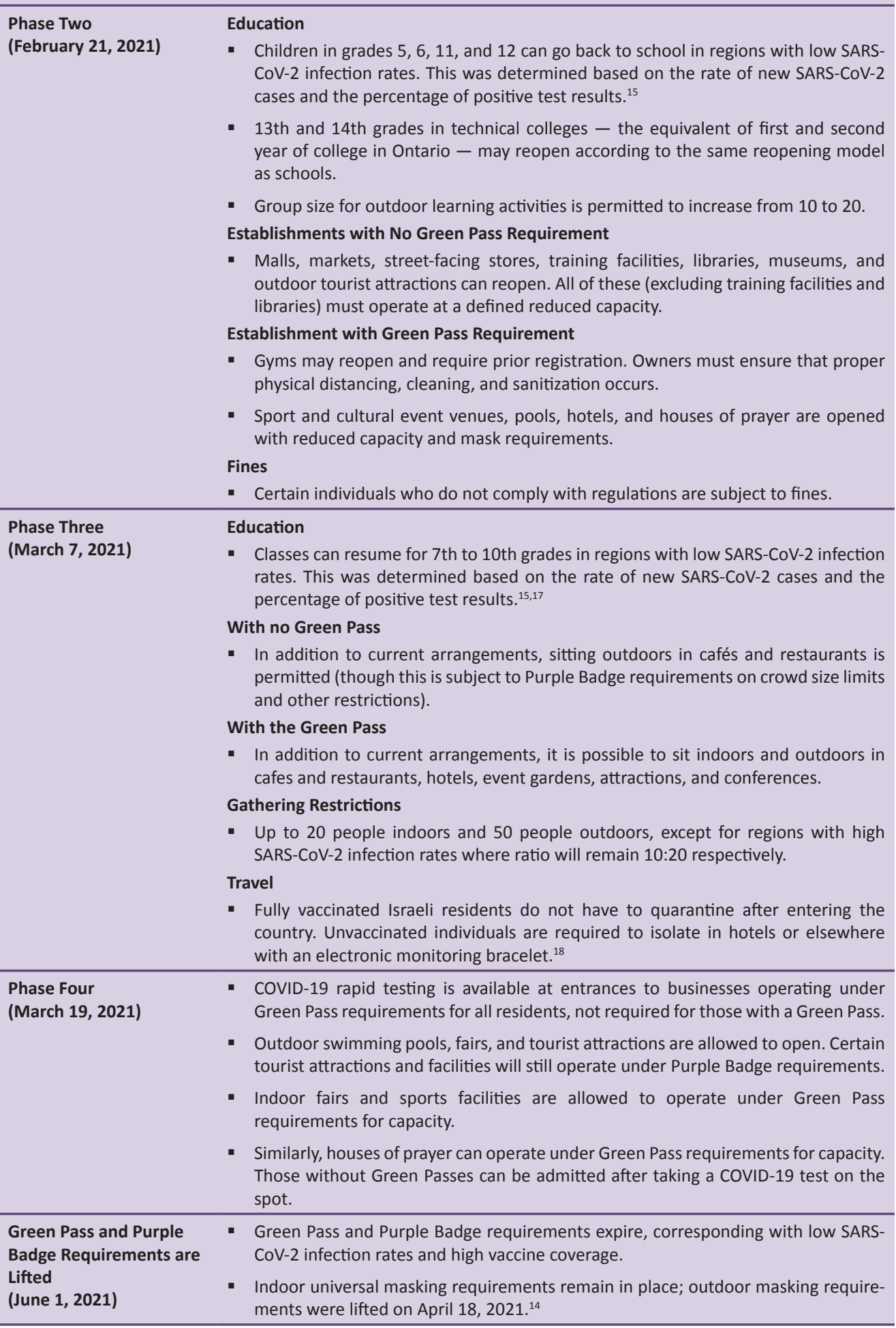

Table 1. Stages of Israel's Phased Reopening

Table describing the key components of each stage of Israel's phased reopening, which began on February 7, 2021, and progressed through three additional stages. Information sourced from the Israel Ministry of Health. ${ }^{19,20,21,22}$

Table 2 describes the necessary steps to apply for and present a Green Pass, as well as current establishments in Israel that require a Green Pass. 


\begin{tabular}{|c|c|}
\hline Eligible to Apply & $\begin{array}{l}\text { - Individuals who are fully vaccinated or considered recovered from } \\
\text { COVID-19. Vaccination is available to those } 16 \text { and older, regardless of } \\
\text { healthcare coverage. } \\
\text { - Individuals } 16 \text { and younger who tested negative in a COVID-19 PCR test. } \\
\text { They will receive a Green Pass that is valid for } 72 \text { hours from their day of } \\
\text { testing. }\end{array}$ \\
\hline Application Process & $\begin{array}{l}\text { - Options to apply online, on an app, on the Ministry of Health Hotline, or } \\
\text { with interactive voice response. } \\
\text { - Personal information is passed through a secure central repository that } \\
\text { informs you if you are fully vaccinated, recovered, or a confirmed case. }\end{array}$ \\
\hline $\begin{array}{l}\text { Current Establishments with } \\
\text { Green Pass Requirements }\end{array}$ & - Sports and cultural event venues, concert halls. \\
\hline $\begin{array}{l}\text { Presentation of Green Pass and } \\
\text { Pass } \\
\text { Expiration }\end{array}$ & $\begin{array}{l}\text { - Establishments with Green Pass requirements are required to place a sign } \\
\text { informing visitors of Green Pass requirements. } \\
\text { - Individuals must present the Green Pass - using a QR code on the Minis- } \\
\text { try of Health 'Traffic Light App' or a printed copy - and photo I.D. when } \\
\text { entering an establishment requiring a Green Pass. } \\
\text { - Green Pass requirements expire as of June } 1,2021 \text {. } \\
\text { - Vaccination certificate or certificate of recovery (granted without an ap- } \\
\text { plication) can replace the Green Pass, but the Green Pass is preferable. }\end{array}$ \\
\hline
\end{tabular}

Table 2. Israel's Green Pass (Introduced on February 21, 2021)

Table describing the process of obtaining and presenting a Green Pass, establishments requiring a Green Pass, and Green Pass expiration. Since Phase 4 of reopening, access to establishments without a Green Pass has greatly expanded; most establishments must meet the Purple Badge standard (see below). Information sourced from the Israel Ministry of Health. ${ }^{23}$

\section{Requirements to Meet the Purple Badge Standard}

Retail stores and non-essential industries and workplaces were permitted to reopen - with or without a Green Pass - provided that they agreed to follow a set of public health measures to meet the Purple Badge standard. ${ }^{24}$ These establishments included restaurants, gyms, pools, stores selling cultural and leisure items, and other businesses. The Purple Badge requirements officially expired on June 1, 2021.

Employers were required to appoint an employee to be responsible for monitoring and managing these measures. For all workplaces, there was mandatory COVID-19 screening prior to entry of employees and customers. This process was comprised of a non-invasive temperature check and screening questions about potential COVID-19 symptoms and recent close contact with COVID-19 patients. Additional signage and measures to maintain a distance of at least 2 metres between individuals of different households was also required in businesses and workplaces. If this was not possible, there had to still be continuous enforcement of measures to prevent infection. Sharing equipment and workspaces should have been avoided, and cohorting groups of workers was required. Employees who could work from home and conduct their work outside the workplace setting were required to do so to reduce capacity.

All workplaces had to operate at a defined reduced capacity - most often determined using the area of an establishment - and the employer and individual responsible for upholding the Purple Badge rules must sign a document committing to implement these rules. If there is one or more SARS-CoV-2 case in a non-essential workplace or industry, the district's medical officer of health or the business owner can order the full or partial closure of the establishment. The Ministry of Health may conduct an investigation and specify subsequent reopening steps. 
Impact of Israel's Phased Reopening During COVID-19 Vaccine Rollout on SARSCoV-2 Cases and COVID-19 Hospitalizations, ICU Admissions, and Deaths

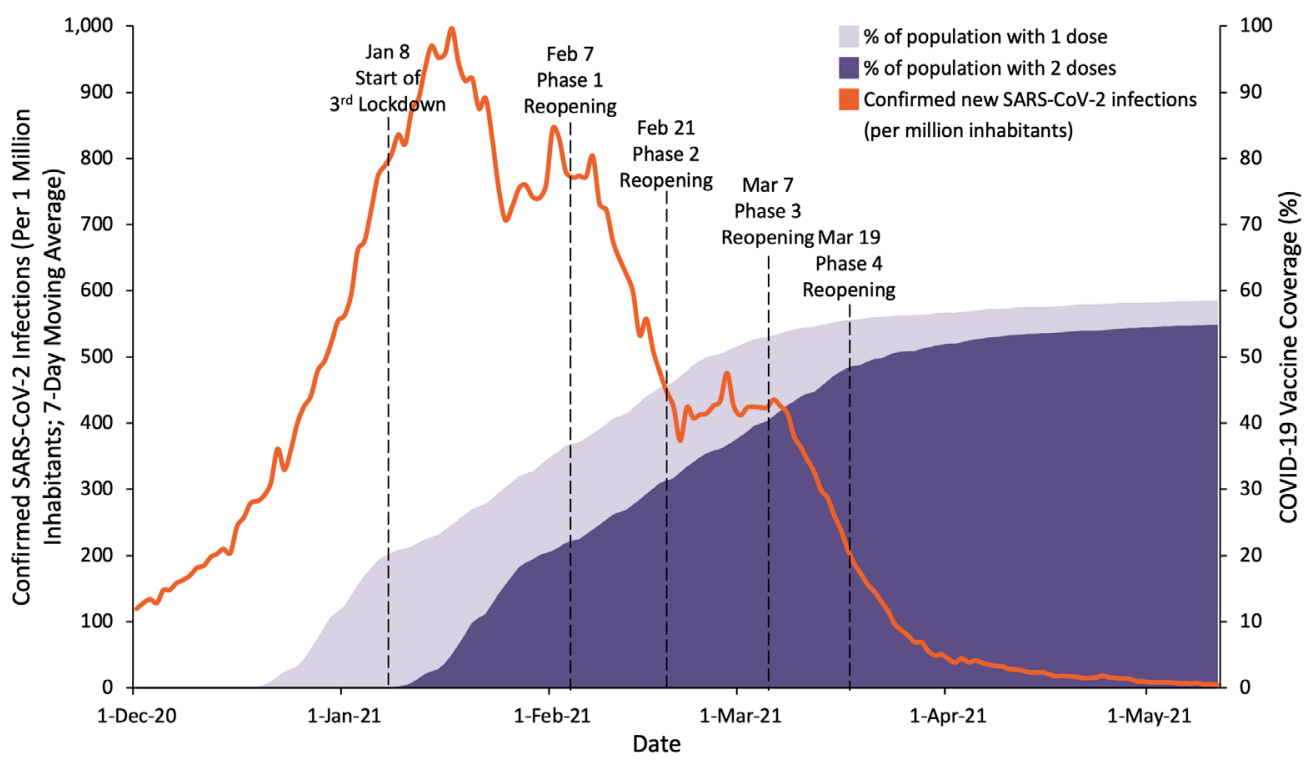

Figure 1. Confirmed New SARS-CoV-2 Infections and COVID-19 Vaccine Coverage During Israel's Phased Reopening Line graph presenting the 7-day moving average of confirmed new SARS-CoV-2 infections per million inhabitants from December 1, 2020, to May 11, 2021. The lighter purple shaded area shows the percentage of Israelis of any age who have received one dose of a COVID-19 vaccine, and the darker purple shaded area shows the percentage that have received two doses. The dashed lines mark the starting dates of Israel's third lockdown, which ended on February 7 , 2021, and each phase of Israel's reopening. Data sourced from Our World in Data and the Israel Ministry of Health. ${ }^{25,26}$

Between February 7, 2021 - the start of the first phase of reopening-and March 7, 2021-the start of the third phase of reopening - there was a $47.1 \%$ drop in SARSCoV-2 cases. As well, first and second dose COVID-19 vaccine coverage went from $38.2 \%$ and $23.8 \%$ to $53.5 \%$ and $41.71 \%$, respectively. Following the third phase of reopening there was a significant decline in SARS-CoV-2 cases. By April 19, 2021, the 7-day moving average was only 17.5 SARS-CoV-2 cases per million inhabitants, indicating effective control of the pandemic in Israel. ${ }^{27}$ As seen in Figure 1, after the first three phases of reopening, there was a net decline in SARS-CoV-2 cases over subsequent weeks as COVID-19 vaccine coverage continued to increase.

Although there was some decline in the 7-day moving average of SARS-CoV-2 cases following the implementation of the nationwide lockdown, sharper declines occurred following increased COVID-19 vaccine uptake. Steeper reductions also occurred earlier in people aged 65 years and above, and around 3-4 weeks later in people aged 16 to 24 years, which corresponds to when COVID-19 vaccine coverage in this group began to increase. ${ }^{28}$ 


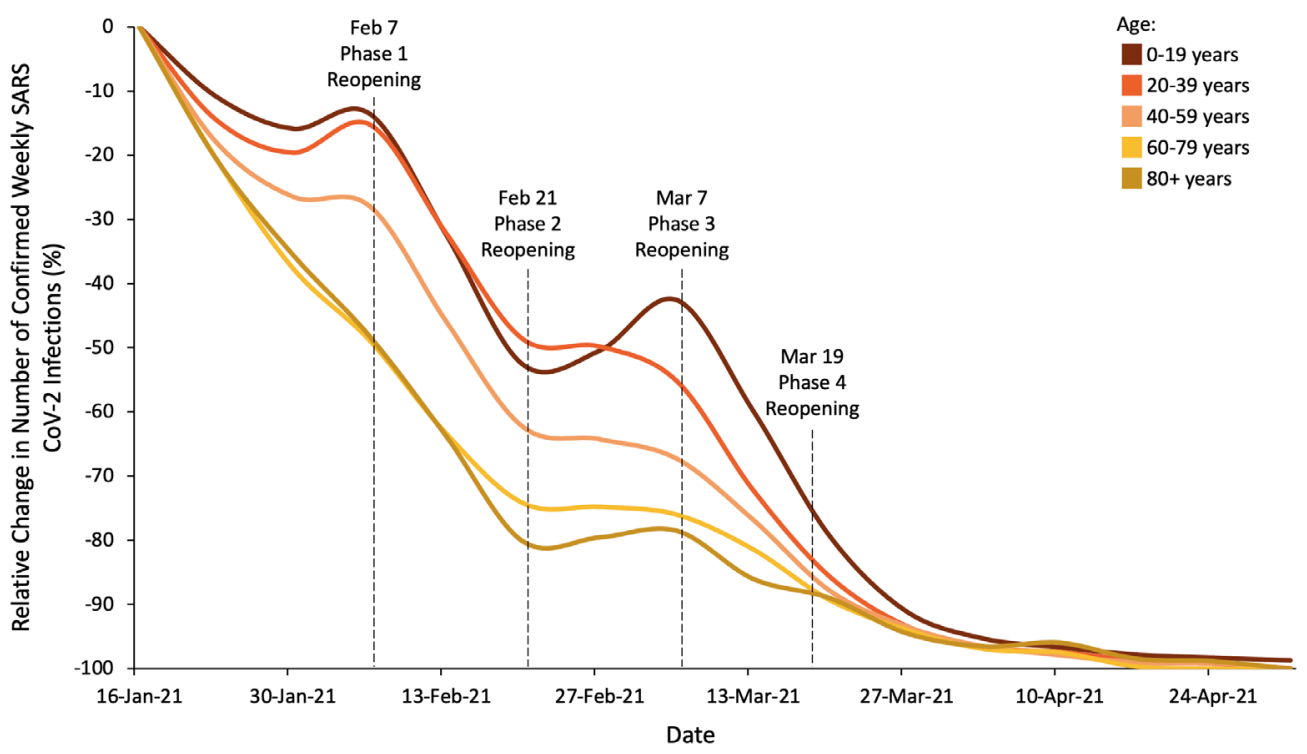

Figure 2. Relative Change in Number of Confirmed Weekly SARS-CoV-2 Infections by Age Group During Israel's Phased Reopening

Line graph presenting the relative change, as a percentage, of confirmed weekly SARS-CoV-2 infections by age group from the week of January 16, 2021, to the week of May 1, 2021. The relative change for all age groups was indexed using the number of confirmed weekly SARS-CoV-2 cases at the peak of Israel's third wave in January 2021. The dashed lines mark the starting dates of each phase of Israel's reopening. Data sourced from Our World in Data and the Government of Israel. ${ }^{8}$

Although individuals younger than 16 years of age have yet to be vaccinated in Israel, Figure 2 demonstrates that Israel's national vaccination campaign also resulted in a marked decrease in the number of confirmed SARS-CoV-2 infections in 0 to 19-yearolds, even during phased reopening of school and daycare settings.

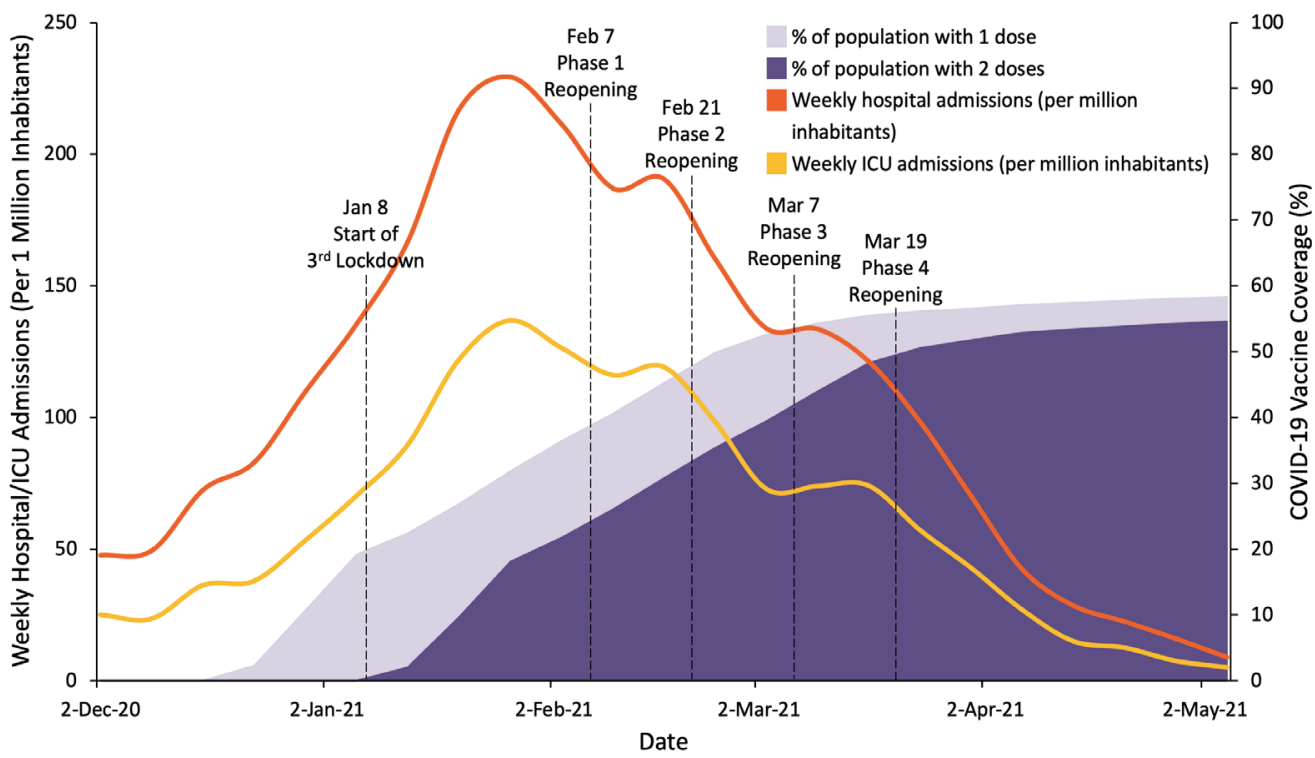

Figure 3. Weekly Hospital and ICU Admissions and COVID-19 Vaccine Coverage During Israel's Phased Reopening Line graph presenting the weekly hospital and intensive care unit (ICU) admissions per million inhabitants from the week of December 2, 2020, to the week of May 2, 2021. The lighter purple shaded area shows the percentage of Israelis of any age who have received one dose of a COVID-19 vaccine, and the darker purple shaded area shows the percentage that have received two doses. The dashed lines mark the starting dates of Israel's third lockdown, which ended on February 7, 2021, and each phase of Israel's reopening. Data sourced from Our World in Data and the Israel Ministry of Health. ${ }^{25,26}$

The weekly COVID-19 hospital and ICU admissions in Israel continued to decline throughout all reopening phases. In parallel to the observed trend in Figure 1, hospitalizations and ICU admissions decreased slightly during Israel's lockdown, continued to decline throughout the first two reopening phases, and decreased 
substantially throughout the remainder of reopening.

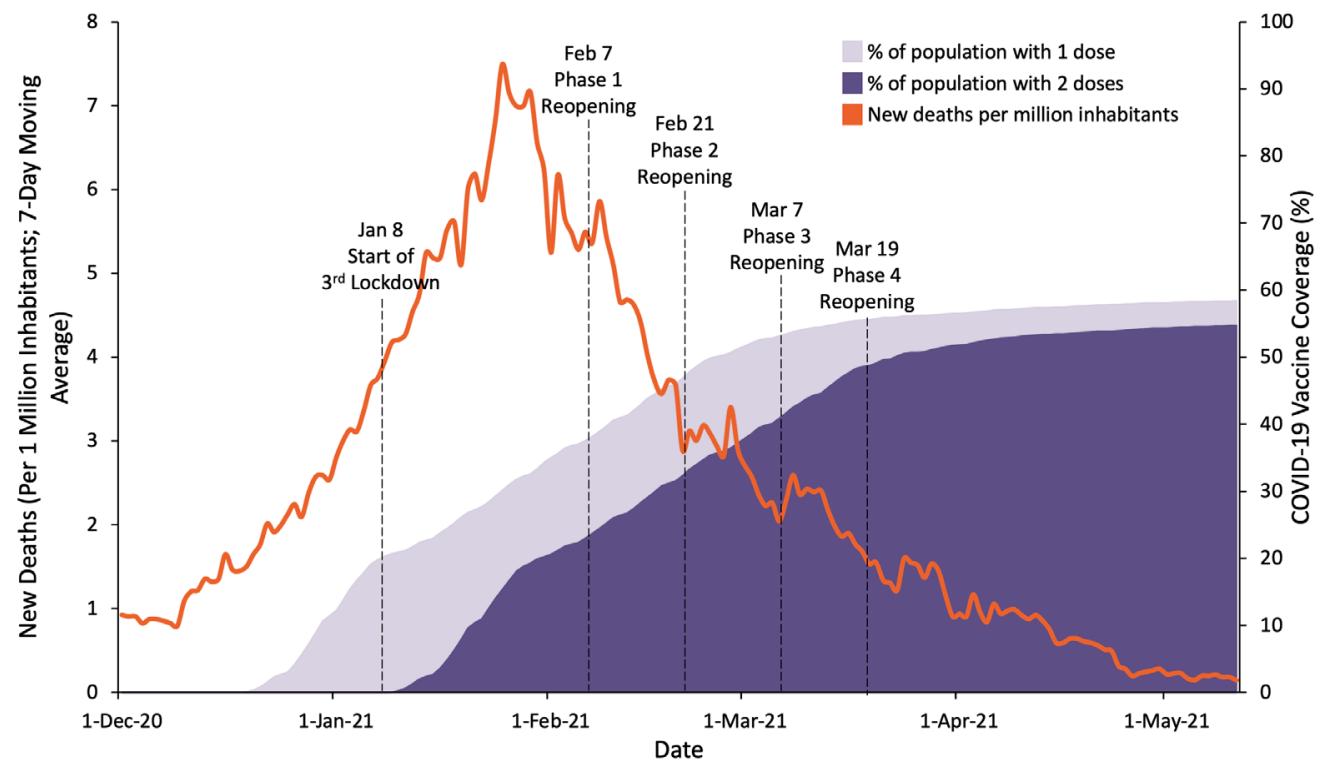

Figure 4. New Deaths and COVID-19 Vaccine Coverage During Israel's Phased Reopening

Line graph presenting the 7-day moving average of new deaths per million inhabitants from December 1, 2020 to May 1, 2021. The lighter purple shaded area shows the percentage of Israelis of any age who have received one dose of a COVID-19 vaccine, and the darker purple shaded area shows the percentage that have received two doses. The dashed lines mark the starting dates of Israel's third lockdown, which ended on February 7, 2021, and each phase of Israel's reopening. Data sourced from Our World in Data and the Israel Ministry of Health. ${ }^{25,26}$

In Figure 4, the 7-day moving average of deaths per million inhabitants begins to decrease approximately two weeks before phase 1 of reopening, during the lockdown, when first and second dose COVID-19 vaccine coverage was $30.1 \%$ and $15.4 \%$, respectively.

\section{Ontario's Current SARS-CoV-2 Infection Rate and COVID-19 Vaccination Coverage Compared to Israel's at the Start of its Phased Reopening}

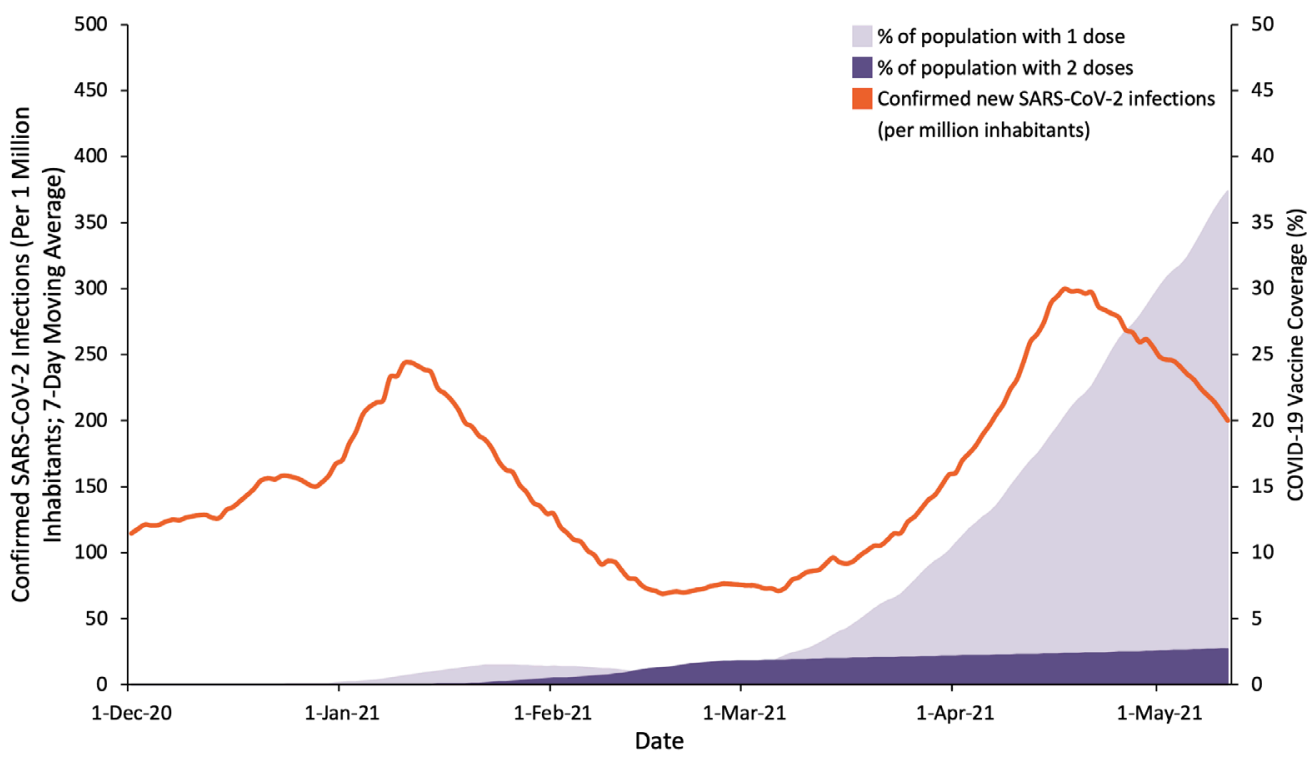

Figure 5. Confirmed New SARS-CoV-2 Infections and COVID-19 Vaccine Coverage in Ontario

Line graph presenting the 7-day moving average of confirmed SARS-CoV-2 infections per million inhabitants in Ontario from December 1, 2020 to May 11, 2021. The lighter purple shaded area shows the percentage of Ontario residents of any age who have received one dose of a COVID-19 vaccine, and the darker purple shaded area shows the percentage that have received two doses. The population of Ontario was assumed to be steady at 14,567,815 throughout the entire observation period. SARS-CoV-2 cases are presented as the number of new SARS-CoV-2 cases per 1,000,000 individuals reported on May 11. Data sourced from the Government of Ontario. 2,29

Ontario's 7-day moving average of confirmed new SARS-CoV-2 infections during its third wave has begun to decline after reaching a peak of approximately 300.0 confirmed 
cases per million individuals on April 17, 2021. Ontario's 7-day moving average of new SARS-CoV-2 cases was lower than Israel's at each of their reopening phases. As of May 11, 2021, the final date shown in Figure 5, 38.1\% of Ontario's population were vaccinated with one dose of COVID-19 vaccine, and $2.7 \%$ were vaccinated with two doses. The coverage for the first dose on this date is nearly identical to Israel's first dose vaccine coverage at the start of its first phase of reopening; however, Ontario's coverage for the second dose is considerably lower than what Israel had during its first stage of reopening (23.8\%).

\section{Interpretation}

The pace and speed of Israel and Canada's COVID-19 vaccination efforts differ substantially due to vaccine supply and access issues. Israel offered the administration of a second COVID-19 vaccine dose approximately 21 days after the first dose. However, in Canada, the interval from first to second dose of COVID-19 vaccines was extended up to four months due to limited supply.

Israel executed a phased reopening in conjunction with a national COVID-19 vaccination campaign, with sustained decreases in SARS-CoV-2 infections and COVID-19 hospitalization, ICU admissions, and deaths throughout. There are several key takeaways from Israel's successful approach that can inform reopening efforts in Ontario.

Notably, as seen in Table 1, the first phase of Israel's reopening was of outdoor spaces, directly followed by the reopening of schools, staggered by ages with the youngest students returning first. Opening of indoor establishments and loosening of restrictions on indoor gatherings were among the final stages of reopening. Throughout Israel's reopening phases and vaccination campaign, there has been continued enforcement of public health measures with an indoor masking policy in public spaces and recommendations of physical distancing and proper hand sanitization.

Israel also continued to aggressively test, trace, and isolate. To further limit the spread of COVID-19, Israel has open SARS-CoV-2 testing for all residents - including asymptomatic individuals - and conducts contact tracing for all cases. ${ }^{30}$ Israel did this for the entirety of the third pandemic wave, and all samples are sequenced to test for VOCs. Israel continues to exercise strict travel controls, currently banning outbound travel to seven countries with widespread presence of VOCs. ${ }^{31}$ Returning travellers from abroad are also required to present a COVID-19 recovery certificate, a vaccination certificate, serology testing, or a negative PCR test results from within 72 hours prior to air travel.

The Green Pass, a vaccination certificate or passport, was designed to protect highrisk vaccinated individuals and allow them to participate in indoor activities at a time of continued community spread of SARS-CoV-2 - since these individuals (older multimorbid patients) have lower vaccine effectiveness. ${ }^{10,11}$

The Green Pass approach also had a notable secondary impact to motivate citizens to get fully vaccinated by granting pass holders access to socially desirable cultural venues, sports events, and concert halls. When SARS-CoV-2 infection levels were higher, restaurants, hotels, gyms and other institutions also required a Green Pass to enter.

While there is no available data, the Green Pass may have also helped reduce SARSCoV-2 transmission in higher-risk settings. Israel's Purple Badge standard ensures that customers, employees, and employers in non-essential workplaces and retail stores can operate safely and efficiently without customers needing to show proof of vaccination or COVID-19 recovery.

Green Pass and Purple Badge standard requirements were officially removed in Israel on June 1, 2021 - corresponding with low nationwide SARS-CoV-2 infection numbers 
(only 17 new cases on May 31, 2021) and high COVID-19 vaccine coverage. The only remaining public health measure is indoor masking. Both the Green Pass and Purple Badge standard were key initiatives to incentivize COVID-19 vaccine uptake and facilitate Israel's safe reopening.

Various aspects contributed to Israel's vaccine rollout and uptake success, including: the country's simple prioritization process, effective communication to instill vaccine confidence, digital infrastructure and penetration of smartphone apps and its small geographical area and population size..$^{32,33}$

There are important concerns related to equity and privacy with respect to Israel's Green Pass approach. Firstly, while this strategy was primarily designed to protect high-risk individuals, it also incentivized vaccines for groups who are hesitant. It can theoretically exacerbate existing social inequities - although this hindrance can be overcome as shown in Israel with relatively high eventual uptake in all population subgroups.

While the Green Pass approach could be intended to promote COVID-19 vaccine uptake, it may not address or incentivize vaccinations for those who lack vaccine confidence. Those with limited access to technological devices may have faced barriers when applying for a Green Pass. Community engagement, trust building and targeted information are needed to address concerns of groups who are vaccine hesitant. Privacy concerns have also been noted with the Green Pass insofar as those checking credentials have access to private health information, albeit just limited to vaccination status. ${ }^{34}$

At entrances to businesses that operated under Green Pass requirements, rapid COVID-19 tests could be used upon entry for all residents, but not required for those with a Green Pass - addressing much of the equitable access issue.

Although Israel has only vaccinated residents 16 years of age and older, those younger than age 16 similarly experienced a substantial drop in SARS-CoV-2 infected cases (see Figure 2) during Israel's national vaccination campaign. A similar trend was observed in the United Kingdom. ${ }^{35}$ Israel has mandated mask-wearing in schools and requires masks in indoor public spaces. Children also cannot participate in cultural events, sports events, and concerts without having had a negative COVID-19 PCR test within 72 hours prior to participating.

Applying Israel's phased reopening strategy, aligned with improving epidemiological indicators, increasing vaccine uptake and maintaining key public health measures could inform Ontario's approach. In Israel, this approach showed declines in SARSCoV-2 cases, and COVID-19 hospitalization and deaths. Other promising practices to consider include the Green Pass approach which can incentivize vaccine uptake, emphasize outdoor activities and support a safe return to social and communal events in the arts and recreation.

The Purple Badge approach, which indicated workplace safety standards, could also support a safe reopening of Ontario workplaces, which have experienced a significant number of cases and outbreaks. However, it is important to consider some of the key differences between Israel and Ontario. Ontario has a population 1.6 times greater than Israel, but with 45 times the land area; ${ }^{37-39}$ as well, vaccine supply and access, digital infrastructure (centralized in Israel), and the timing of the second dose (with Israel insisting on 2-doses per protocol within 3 weeks) all helped to enable a robust and rapid vaccination campaign and reopening strategy in Israel.

Examining Israel's reopening is instructive given the phased approach, short timeline, very high number of fully vaccinated individuals, and absence of a surge in SARS-CoV-2 cases following the lifting of restrictions. As Ontario continues to plan and implement its own reopening strategy, examining the reopening of other jurisdictions is important. This includes the United Kingdom, which, like Ontario, has a diverse population, saw 
essential workers and racialized groups disproportionately impacted by the COVID-19 pandemic, ${ }^{40}$ and maximized first COVID-19 vaccine doses by extending the dosing interval by several weeks.

\section{Methods Used for This Science Brief}

Data used in the analysis of Israel's SARS-CoV-2 cases, COVID-19 hospitalizations, ICU admissions and deaths as well as COVID-19 vaccination was sourced from Our World in Data and the Israel Ministry of Health and was current as of May 11, 2021.9,19,20 Data used in the analysis of Ontario's SARS-CoV-2 cases, COVID-19 hospitalizations, ICU admissions and deaths as well as COVID-19 vaccination was sourced from the Ontario Ministry of Health and was current as of May 11, 2021.2,29

\section{Author Contributions}

GMK and NMS conceived the Science Brief. GMK, KBB, and NMS wrote the first draft of the Science Brief. GMK performed the analyses. All authors revised the Science Brief critically for important intellectual content and approved the final version.

\section{References}

1. Tuite AR, Fisman DN, Odutayo A, et al. COVID-19 hospitalizations, ICU admissions and deaths associated with the new variants of concern. Science Briefs of the Ontario COVID-19 Science Advisory Table. 2021;1(18). https://doi.org/10.47326/ ocsat.2021.02.18.1.0

2. Government of Ontario. All Ontario: Case numbers and spread. Ontario.ca. Accessed May 21, 2021. https://covid-19.ontario.ca/data

3. Government of Ontario. Hospitalizations. Ontario.ca. Accessed May 14, 2021. https://covid-19.ontario.ca/data/hospitalizations

4. Ontario Vaccination Data. COVID-19 Tracker Canada. Accessed May 21, 2021. https://covid19tracker.ca/provincevac.html?p=ON

5. Ontario Releases Three-Step Roadmap to Safely Reopen the Province. news. ontario.ca. Accessed May 21, 2021. https://news.ontario.ca/en/release/1000161/ ontario-releases-three-step-roadmap-to-safely-reopen-the-province

6. Public Health Agency of Canada. Update on COVID-19 in Canada: Epidemiology and Modelling. Canada.ca. Accessed May 14, 2021. https://www.canada.ca/ content/dam/phac-aspc/documents/services/diseases-maladies/coronavirusdisease-covid-19/epidemiological-economic-research-data/update-covid-19canada-epidemiology-modelling-20210423-en.pdf

7. Hannah R, Ortiz-Ospina E, Beltekian D, et al. Coronavirus (COVID-19) Vaccinations. Our World in Data. Published January 21, 2021. Accessed January 21, 2021. https:// ourworldindata.org/covid-vaccinations

8. Mathieu E, Ritchie H. Vaccinations and the impact of COVID-19- our continuouslyupdated data for Israel. Our World in Data. Accessed May 21, 2021. https:// ourworldindata.org/vaccination-israel-impact

9. Williams D, Lubell M. More than half Israelis receive both COVID-19 vaccine doses. Reuters. Accessed May 21, 2021. https://www.reuters.com/article/us-healthcoronavirus-israel-vaccine-idUSKBN2BHOHO

10. Barda N, Dagan N, Balicer RD. BNT162b2 mRNA Covid-19 Vaccine in a Nationwide Mass Vaccination Setting. Reply. N Engl J Med. 2021;384(20):1970. https://doi. org/10.1056/NEJMc2104281 
11. Dagan N, Barda N, Kepten E, et al. BNT162b2 mRNA Covid-19 Vaccine in a Nationwide Mass Vaccination Setting. N Engl J Med. 2021;384(15):1412-1423. https://doi.org/10.1056/NEJMoa2101765

12. Wilf-Miron R, Myers V, Saban M. Incentivizing Vaccination Uptake: The "Green Pass" Proposal in Israel. JAMA. 2021;325(15):1503. https://doi.org/10.1001/ jama.2021.4300

13. Joint Prime Minister's Office-Health Ministry-Finance Ministry statement 7 January 2021. Israel Ministry of Foreign Affairs. Published January 7, 2021. Accessed May 28, 2021. https://mfa.gov.il/MFA/Innovativelsrael/Economy/Pages/Joint-PrimeMinister-s-Office-Health-Ministry-Finance-Ministry-statement-7-January-2021. aspx

14. Israel Ministry of Health. Effective Sunday, 18.4: Wearing a Mask Outdoors Is No Longer Mandatory. GOV.IL. Published April 17, 2021. Accessed May 28, 2021. https://www.gov.il/en/departments/news/17042021-01

15. Local Councils Traffic Light Model. Corona Traffic Light Model (Ramzor) Website. Accessed May 14, 2021. https://corona.health.gov.il/en/local-councils-trafficlight-model/

16. TOI Staff. Some Israeli kids head back to school amid confusion and uncertainty. The Times of Israel. Published February 11, 2021. Accessed May 30, 2021. https://www.timesofisrael.com/some-israel-kids-heading-back-to-school-amidconfusion-and-uncertainty/

17. TOI Staff. Mayors push plan to reopen all school grades by March 1; health minister opposed. The Times of Israel. Published February 22, 2021. Accessed May 30, 2021. https://www.timesofisrael.com/mayors-push-plan-to-reopen-allschool-grades-by-march-1-health-minister-opposed/

18. Israel. COVID-19 Health System Response Monitor. Accessed May 21, 2021. https://www.covid19healthsystem.org/countries/israel/countrypage.aspx

19. Government of Israel. The Lockdown in its Current Format Has Been Extended until Sunday, 7.2.2021 at 07:00 AM. GOV.IL. Accessed May 14, 2021. https://www. gov.il/en/Departments/news/05022021-01

20. Government of Israel. Second Phase Restrictions on Reopening to Take Effect on Sunday, 21.2.2021. GOV.IL. Accessed May 14, 2021. https://www.gov.il/en/ Departments/news/19022021-03

21. Government of Israel. Government has Approved the Third Reopening Phase as Planned, Effective 7.3.2021. GOV.IL. Accessed May 14, 2021. https://www.gov.il/ en/departments/news/01032021-03

22. Government of Israel. Regulations for the Fourth Phase of the Reopening Program Approved, Effective 19.3.2021. GOV.IL. Accessed May 14, 2021. https://www.gov. il/en/Departments/news/18032021-03

23. Israel Ministry of Health. What is a Green Pass? Corona Traffic Light Model (Ramzor) Website. Accessed May 21, 2021. https://corona.health.gov.il/en/ directives/green-pass-info/

24. Government of Israel. The "purple character" - the economy is moving to a routine in the shadow of the corona. GOV.IL. Accessed May 21, 2021. https://www.gov.il/ he/departments/guides/purple_note

25. Roser M, Ritchie H. Coronavirus Pandemic (COVID-19). Our World in Data. Published online March 4, 2020. https://ourworldindata.org/coronavirus

26. Corona - control panel. Israel Ministry of Health. Accessed May 22, 2021. https:// 
datadashboard.health.gov.il/COVID-19/general

27. Leshem $E$, Wilder-Smith A. COVID-19 vaccine impact in Israel and a way out of the pandemic. The Lancet. 2021;397(10287):1783-1785. https://doi.org/10.1016/ S0140-6736(21)01018-7

28. Haas EJ, Angulo FJ, McLaughlin JM, et al. Impact and effectiveness of mRNA BNT162b2 vaccine against SARS-CoV-2 infections and COVID-19 cases, hospitalisations, and deaths following a nationwide vaccination campaign in Israel: an observational study using national surveillance data. The Lancet. 2021;0(0). https://doi.org/10.1016/S0140-6736(21)00947-8

29. Government of Ontario. COVID-19 Vaccine Data | Données sur les vaccins contre la COVID-19 - Ontario Data Catalogue. Ontario.ca. Accessed May 11, 2021. https:// data.ontario.ca/dataset/covid-19-vaccine-data-in-ontario/resource/8a89caa9511c-4568-af89-7f2174b4378c

30. Coronavirus (COVID-19) Testing - Statistics and Research. Our World in Data. Accessed May 21, 2021. https://ourworldindata.org/coronavirus-testing

31. Air travel to and from Israel. GOV.IL. Accessed May 21, 2021. https://www.gov.il/ en/Departments/Guides/flying-to-israel-guidlines?chapterIndex $=2$

32. Choi Y, Stall NM, Maltsev A, et al. Lessons learned from Israel's vaccine rollout. Science Briefs of the Ontario COVID-19 Science Advisory Table. 2021;2(9). https:// doi.org/10.47326/ocsat.2021.02.09.1.0

33. Balicer RD, Ohana R. Israel's COVID-19 endgame. Science. 2021;372(6543):663663. https://doi.org/10.1126/science.abj3858

34. Hall MA, Studdert DM. "Vaccine Passport" Certification - Policy and Ethical Considerations. New England Journal of Medicine. Published online March 31, 2021. https://doi.org/10.1056/NEJMp2104289

35. Public Health England. National flu and COVID-19 surveillance graph: 27 May 2021 (week 21). GOV.UK. Published May 27, 2021. Accessed May 28, 2021. https://www. gov.uk/government/statistics/national-flu-and-covid-19-surveillance-reports

36. Mishra S, Stall NM, Ma H, et al. A vaccination strategy for Ontario COVID-19 hotspots and essential workers. Science Briefs of the Ontario COVID-19 Science Advisory Table. 2021;2(26). https://doi.org/10.47326/ocsat.2021.02.26.1.0

37. Population, 1800 to 2019. Our World in Data. Published 2019. Accessed May 30, 2021. https://ourworldindata.org/grapher/population

38. Land area. Our World in Data. Published 2016. Accessed May 28, 2021. https:// ourworldindata.org/grapher/land-area-km

39. Geoffrey E, Michiel H, Wise SF. Ontario. Encyclopedia Britannica. Accessed May 30, 2021. https://www.britannica.com/place/Ontario-province

40. Razak F, Slutsky A, Naylor D. Can we follow the British model to tame the virus by Canada Day? The Globe and Mail. https://www.theglobeandmail.com/opinion/ article-can-we-follow-the-british-model-to-tame-the-virus-by-canada-day/. Published April 29, 2021. Accessed May 30, 2021. 